\title{
Differential expression of tumor necrosis factor $\alpha$, interleukin $1 \beta$, nuclear factor $\kappa B$ in nasal mucosa among chronic rhinosinusitis patients with and without polyps
}

\author{
Danuta Plewka ${ }^{1}$, Alicja Grzanka², Elzbieta Drzewiecka, Andrzej Plewka3, Maciej Misiołek ${ }^{4}$, Grażyna Lisowska \\ Beata Rostkowska-Nadolska ${ }^{5}$, Radoslaw Gawlik ${ }^{6}$
}

\author{
'Department of Cytophysiology, Chair of Histology and Embryology, Medical University of Silesia, Katowice, Poland \\ 2Department of Internal Medicine, Dermatology and Allergology, Medical University of Silesia, Katowice, Poland \\ ${ }^{3}$ Department of Proteomics, Medical University of Silesia, Katowice, Poland \\ ${ }^{4}$ Department of Otolaryngology in Zabrze, Medical University of Silesia in Katowice, Poland \\ ${ }^{5}$ Department of Otolaryngology, Medical University of Wroclaw, Wroclaw, Poland \\ ${ }^{6}$ Department of Internal Diseases, Allergology and Clinical Immunology, Medical University of Silesia, Katowice, Poland \\ Adv Dermatol Allergol 2017; XXXIV (3): 199-206 \\ DOI: https://doi.org/10.5114/ada.2017.67842
}

\begin{abstract}
Introduction: The pathogenesis of nasal polyps is still not fully understood.

Aim: To analyze the topography and intensity of interleukin $1 \beta$ (IL-1 $\beta$ ), tumor necrosis factor $\alpha$ (TNF- $\alpha$ ), cyclooxy-

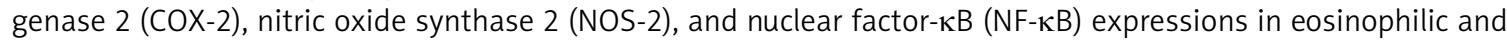
neutrophilic polyps and in normal nasal mucosa.

Material and methods: The study included specimens from 20 patients with eosinophilic polyps (more than 10\% of eosinophils in inflammatory infiltrate), 20 individuals with neutrophilic polyps (predominance of neutrophils and less than $10 \%$ of eosinophils), and samples of normal nasal mucosa from 10 controls. The expressions of studied proteins in vascular endothelial cells, epithelial, stromal and glandular cells were determined immunohistochemically with specific monoclonal antibodies.

Results: Irrespective of the cellular type, the intensity of expressions in eosinophilic and neutrophilic polyps was significantly higher than in the normal mucosa. Eosinophilic polyps were characterized by stronger expressions of TNF- $\alpha$ (in all cellular types), IL-1 $\beta$ (in endothelial, glandular and epithelial cells), NF- $\kappa B$ (in stromal and epithelial cells), COX-2 (in glandular and stromal cells), and NOS-2 (in endothelial and stromal cells). In contrast, neutrophilic polyps showed significantly stronger expressions of COX-2 (in epithelial and endothelial cells) and NOS-2 (in glandular and epithelial cells). In both phenotypes, the strongest expressions of all studied markers were documented in vascular endothelial cells.

Conclusions: Inflammatory markers are involved in pathogenesis of both eosinophilic and neutrophilic polyps. Endothelial defects can play an important role in the development of nasal polyps.
\end{abstract}

Key words: nasal polyp, eosinophil, neutrophil, inflammatory mediators.

\section{Introduction}

The pathogenesis of nasal polyps is still not fully understood. Inflammation of nasal and sinus mucosa, driven by numerous cytokines with a number of cellular subpopulations involved, constitutes a constant component of clinical manifestation in patients with chronic rhinosinusitis with polyps (GRSwNP) [1-3]. Separating tissue of chronic rhinosinusitis patients with (CRSwNP) or without
(CRSsNP) higher eosinophilia was shown in the polypoid form $[1,3]$. However, we still do not know which cells play the vital role in the pathogenesis of polyps. Until recently, the main role in the process of airway mucosa remodeling was ascribed to eosinophils and Th2 skewed response. But patients with chronic rhinosinusitis without polyps have a combined neutrophilic and eosinophilic cellular infiltrate and a mixed Thl/Th2 cytokine profile. However, na-

Address for correspondence: Ass. Prof. Radoslaw Gawlik MD, PhD, Department of Internal Diseases, Allergology and Clinical Immunology, Medical University of Silesia, 35 Ceglana St, 40-952 Katowice, Poland, phone: +48 3235814 35,

e-mail: radwags@poczta.onet.pl

Received: 23.08.2016, accepted: 14.11.2016. 
sal eosinophilia is not observed in patients who developed nasal polyps in the course of cystic fibrosis. Furthermore, patients from Japan, China, and Korea present with the neutrophilic phenotype of polyps markedly more often than individuals from Western countries [4]. However, the neutrophilic phenotype can also represent a secondary form, resulting from bacterial infections associated with polyposis, and local shift from Th2 to Th2/Th17 response. Inflammatory mediators and potential differences in their activity, associated with various inflammatory phenotypes, can play an important role as determinants of the clinical outcome in nasal polyposis. Understanding common and distinct pathophysiological features of various phenotypes of nasal polyps can be helpful in defining the therapeutic targets and implementation of proper treatment. Interleukin $1 \beta$ (IL-1 $\beta$ ) is crucial for host defense responses to infection and injury and tumor necrosis factor $\alpha$ (TNF- $\alpha$ ) is the main cytokine involved in inflammatory and immune response, and their synergy can play a crucial role in the pathogenesis of conditions associated with inflammation, destruction and limited repair of tissues [5]. Inter alia, these cytokines can stimulate an enhanced expression of inflammatory-mediated enzymes, cyclooxygenase 2 (COX-2) and nitric oxide synthase 2 (NOS-2) (the latter previously referred to as inducible nitric oxide synthase). Although the role of nitric oxide in inflammation is complex, its high concentrations and "cross-talk" with COX-2 were shown to enhance inflammatory response $[6,7]$. The COX-2 plays a vital role in autophagy and inflammatory processes induced by IL-1 $\beta$ and TNF- $\alpha$ and in tu-

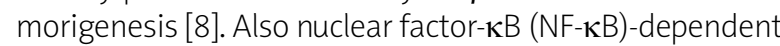
signaling pathways, activated, inter alia, by IL- $1 \beta$ and TNF- $\alpha$ are important for the development of inflammation and tumorigenesis. The NF- $\mathrm{KB}$ is a transcription factor regulating the expression of many genes, whose products modulate inflammation, proliferation, apoptosis, and cellular stress response. Also the genes encoding IL-1 $\beta$, TNF- $\alpha$, COX-2 and NOS-2 are regulated by NF- $\kappa$ B. Activation of the NF- $\kappa$ B pathways induces nasal myofibroblast differentiation and extracellular matrix (ECM) accumulation, what is important in the remodeling process [9].

\section{Aim}

The aim of this prospective study was to analyze the topography and intensity of IL- $1 \beta$, TNF- $\alpha$, COX-2, NOS-2, and NF- $\kappa$ B expressions in tissues of nasal polyps representing various inflammatory phenotypes (eosinophilic vs. neutrophilic) and in nasal mucosa of chronic rhinosinusitis patients.

\section{Material and methods Participants}

The study included 40 patients (20 women and 20 men) aged 26-65 years (mean: $41.6 \pm 9.9$ years), who under- went functional endoscopic sinus surgery (FESS) due to the presence of nasal polyps. None of the patients had a history of previous polypectomy. Two subgroups were differentiated based on the predominant cell type identified on microscopic examination: 20 individuals (11 women and 9 men; mean age: $41.2 \pm 9.7$ years) with eosinophilic nasal polyps (> 10\% of eosinophils in ten high-power fields of hematoxylin and eosin stained slides), and 20 subjects ( 9 women and 11 men; mean age: $42.1 \pm 10.4$ years) with the neutrophilic phenotype of nasal polyps (predominance of neutrophils and $<10 \%$ of eosinophils [2, 3]).

The control group comprised 20 individuals (10 women and 10 men) aged $26-65$ years (mean age: $41.5 \pm 10$ years) with chronic sinusitis without nasal polyps (CRS), who underwent surgery for deviated nasal septum during which tissue sample was obtained. Signs of chronic inflammation were confirmed by the rhinoscopic exam, complete blood count (CBC) and C-reactive protein (CRP) tests performed. Sinus CT were performed to evaluate the sinus pathology excluding patients with abnormal scans.

The exclusion criteria were: the presence of neoplastic disease, cystic fibrosis, anti-asthmatic treatment, positive results of allergic tests, hypersensitivity to aspirin or other non-steroidal anti-inflammatory drugs (NSAIDs), active smoking, pregnancy or lactation, psychiatric treatment, and coagulation disorders. Furthermore, the enrolled patients did not use either topical or systemic glucocorticoids and antihistaminic agents for at least four weeks prior to the polypectomy.

All the subjects provided written consent in accordance with the Declaration of Helsinki. The protocol of the study was approved by the Local Bioethics Committee of the Medical University of Silesia (decision no. NN6501-51/07).

\section{Immunohistochemistry}

Routinely prepared paraffin sections were labeled with rabbit anti-NF-אB (sc-109, Santa Cruz Biotech. Inc., USA), anti-IL-1ß (ab2105, Abcam, Cambridge, USA), antiiNOS (ab15323, Abcam), and anti-TNF- $\alpha$ (ab6671, Abcam) polyclonal antibodies or mouse anti-COX-2 monoclonal antibody (sc-58344, Santa Cruz, USA) as previously described [10]. Digital pictures were taken under magnification 200x, using 20x lens objective Nikon CFI Plan Achromat Series. Numerical aperture was $0.4 \mathrm{~mm}$. The intensity of staining was measured with NIS AR software (Nikon, Japan) as the optical density of the reaction product. Average optical density was calculated for each analyzed area [11]. The isotype controls (the primary antibody was replaced with IgG of some species as the primary antibody) and negative controls (the primary antibody was replaced with antibody diluent) have been done for every studied antigen. We analyzed three sections for every studied protein from every patient, with ten fields examined in each section. All stainings were performed 
by a single person, using exactly the same batch of reagents, in a short period. Finally, the arithmetic mean and standard deviation were calculated.

\section{Statistical analysis}

Normal distribution of the data was verified with the Shapiro-Wilk test. Homogeneity of variance was checked with Levene's test. The values were analyzed via a oneway analysis of variance (one-way Anova). For multiple comparisons, the Games-Howell post-hoc test was used. Results are presented as means \pm standard deviations. $P$-values $<0.05$ were considered significant.

\section{Results}

Cytoplasmic expressions of NF- $\kappa \mathrm{B}, \mathrm{IL}-1 \beta$, TNF- $\alpha$, COX-2, and NOS-2 were documented in vascular endothelial cells, fibroblasts, macrophages, epithelial, stromal and glandular cells, as well as in infiltrating cells, i.e. eosinophils, neutrophils, and monocytes. We analyzed the expressions in vascular endothelial cells, epithelial, stromal and glandular cells of nasal polyps, and chronic rhinosinusitis nasal mucosa.

Irrespective of the cell type, the expressions in polyps representing both inflammatory phenotypes were significantly stronger than in control mucosa. Furthermore, the inflammatory phenotypes of polyps differed in terms of the intensity and topography of analyzed expressions.

\section{Tumor necrosis factor $\alpha$ (TNF- $\alpha$ ) (Figures 1, 2 A)}

\section{Neutrophilic polyps vs. chronic rhinosinusitis}

The expression of TNF- $\alpha$ in the epithelium, glandular tissue and stroma of neutrophilic polyps corresponded to $234 \%, 277 \%$ and $297 \%$ of respective control levels. The expression of TNF- $\alpha$ was the strongest in the vascular endothelium (401\% of the control level).

\section{Eosinophilic polyps vs. chronic rhinosinusitis}

The expression of TNF- $\alpha$ in the epithelium, stroma, and glandular tissue of eosinophilic polyps corresponded to $278 \%, 338 \%$ and $366 \%$ of respective levels documented in chronic rhinosinusitis nasal mucosa. Concentration of TNF- $\alpha$ was the highest in the vascular endothelium (504\% of the control level).

\section{Eosinophilic polyps vs. neutrophilic polyps}

Irrespective of the cell type, the intensity of TNF- $\alpha$ expression in eosinophilic polyps was significantly higher than in neutrophilic polyps.

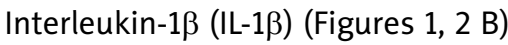

\section{Neutrophilic polyps vs. chronic rhinosinusitis}

The expression of IL-1 $\beta$ in the glandular tissue and epithelium of the neutrophilic polyps corresponded to
$125 \%$ and $146 \%$ of the respective control levels. A markedly stronger expression was documented in the polyp stroma (302\% of the control level). The strongest expression, nearly 4 times greater than in chronic rhinosinusitis nasal mucosa, was observed in the vascular endothelium of polyps.

\section{Eosinophilic polyps vs. chronic rhinosinusitis}

The expression of IL-1 $\beta$ in the glandular tissue, epithelium and stroma of eosinophilic polyps corresponded to $183 \%, 201 \%$, and $396 \%$ of the respective control levels. The highest intensity of IL-1 $\beta$ expression, corresponding to $498 \%$ of the chronic rhinosinusitis mucosal level, was documented in the vascular endothelium of polyps.

\section{Eosinophilic polyps vs. neutrophilic polyps}

The expression of IL-1 $\beta$ in the vascular endothelium, glandular tissue and epithelium of eosinophilic polyps was significantly stronger than in respective cells of neutrophilic polyps. In contrast, the two phenotypes of nasal polyps did not differ significantly in terms of their stromal expressions of IL-1 $\beta$.

\section{Nuclear factor $\kappa \mathrm{B}(\mathrm{NF}-\kappa \mathrm{B})$ (Figures 1, 3)}

\section{Neutrophilic polyps vs. chronic rhinosinusitis}

The expression of NF- $\kappa \mathrm{B}$ in the glandular, stromal, epithelial and endothelial cells of neutrophilic polyps amounted to $170 \%, 227 \%, 296 \%$ and $298 \%$ of the respective control levels.

\section{Eosinophilic polyps vs. chronic rhinosinusitis}

The expression of NF- $\kappa \mathrm{B}$ in the glandular, stromal and epithelial cells, and vascular endothelium of eosinophilic polyps corresponded to $165 \%, 280 \%, 370 \%$ and $384 \%$ of the control levels.

\section{Eosinophilic polyps vs. neutrophilic polyps}

The expressions of NF- $\kappa \mathrm{B}$ in eosinophilic polyps were significantly stronger only in the case of stroma and epithelium. The expression in the other analyzed cell types was not associated with the inflammatory phenotype of nasal polyps.

\section{Cyclooxygenase 2 (COX-2) (Figures 1, 4 A) \\ Neutrophilic polyps vs. chronic rhinosinusitis}

The expression of COX-2 in the glandular tissue, epithelium, stroma and vascular endothelium of neutrophilic polyps corresponded to $288 \%, 300 \%, 359 \%$ and $432 \%$ of the respective control levels.

\section{Eosinophilic polyps vs. chronic rhinosinusitis}

The levels of COX-2 expression in the epithelium, vascular endothelium, glandular tissue and stromal cells of 



Figure 1. Immunohistochemical expression of IL-1 in glands (A-C) of chronic rhinosinusitis nasal mucosa (A), neutrophilic (B) and eosinophilic (C) polyps; of TNF- $\alpha$ in glands (D-F) of CRS nasal mucosa (D), neutrophilic (E) and eosinophilic (F) polyps; of NF-KB in glands (G-I) in CRS nasal mucosa $(\mathrm{G})$, neutrophilic $(\mathrm{H})$ and eosinophilic (I) polyps; of COX-2 in glands $(\mathrm{J}-\mathrm{L})$ of CRS nasal mucosa (J), neutrophilic (K) and eosinophilic (L) polyps, and of NOS-2 in glands (M-O) of CRS nasal mucosa $(M)$, neutrophilic $(N)$ and eosinophilic $(\mathrm{O})$ polyps 

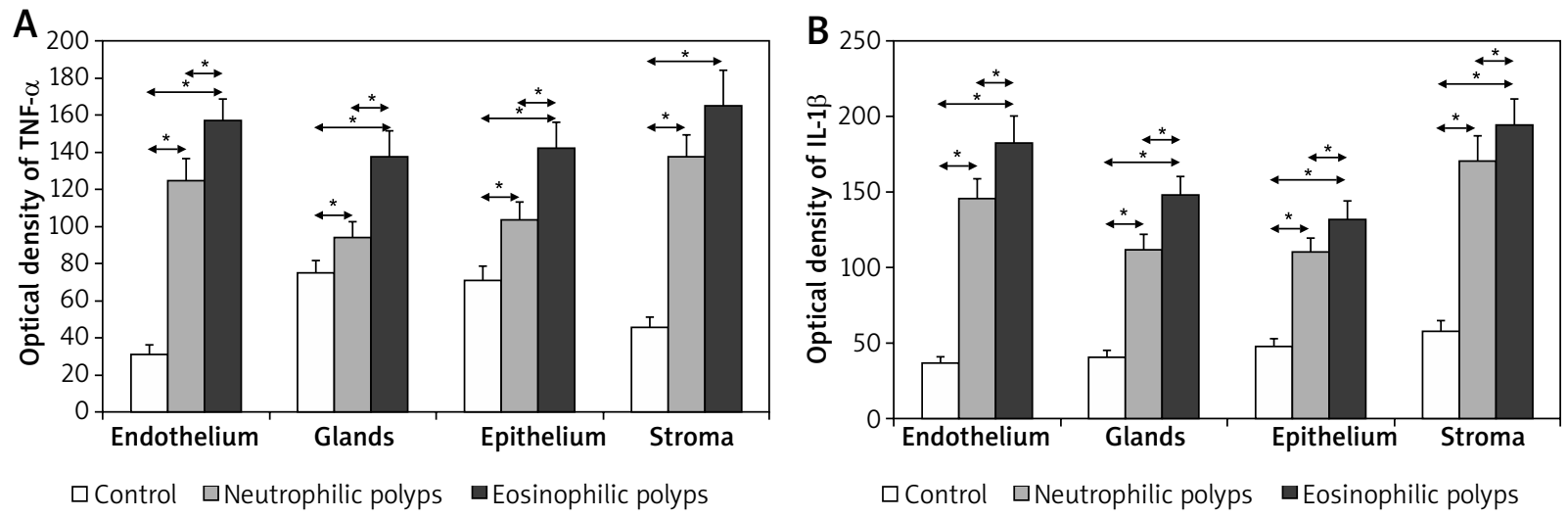

Figure 2. Expression of tumor necrosis factor $\alpha(\mathbf{A})$ and interleukin $1 \beta(B)$ in selected structures of nasal mucosa. Statistically significant differences $(p<0.05)$

eosinophilic polyps amounted to $242 \%, 328 \%, 399 \%$ and $448 \%$ of the respective control levels.

\section{Eosinophilic polyps vs. neutrophilic polyps}

The glandular and stromal expression of COX-2 in eosinophilic polyps was significantly stronger than in neutrophilic polyps. In contrast, neutrophilic polyps showed significantly higher epithelial and endothelial levels of COX-2.

\section{Nitric oxide synthase 2 (NOS-2) (Figures 1, 4 B)}

\section{Neutrophilic polyps vs. chronic rhinosinusitis}

The expressions of NOS-2 in the epithelium, stroma, vascular endothelium and glandular tissue of neutrophilic polyps corresponded to $189 \%, 233 \%, 235 \%$ and $254 \%$ of the respective control levels.

\section{Eosinophilic polyps vs. chronic rhinosinusitis}

The expression of NOS-2 in the epithelial, glandular and stromal cells, and vascular endothelium of eosinophilic polyps amounted to 157\%, 198\%, 282\% and 286\% of the respective control levels.

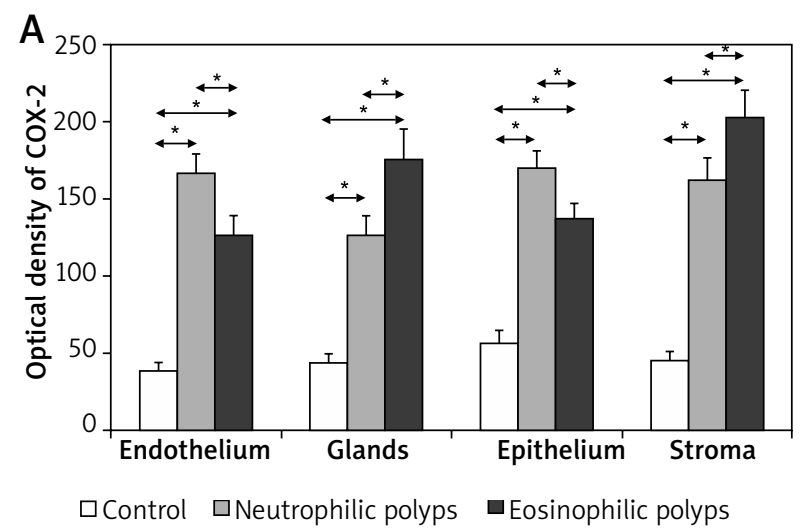

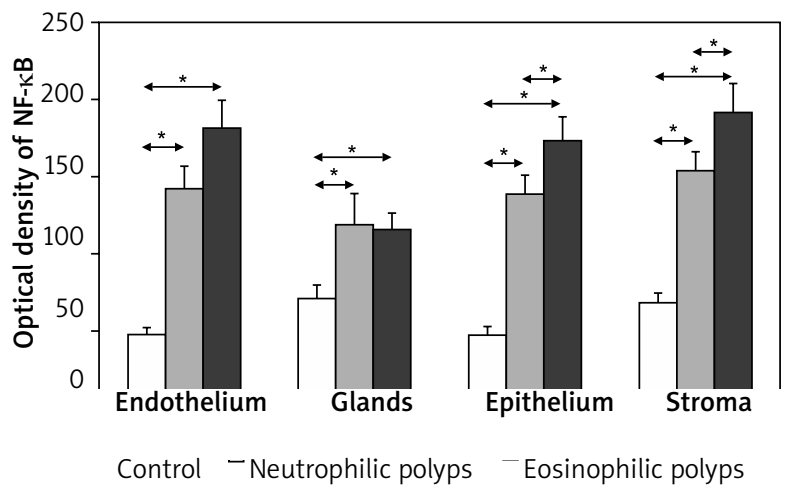

Figure 3. Expression of nuclear factor $\kappa B$ in selected structures of nasal mucosa. Statistically significant differences $(p<0.05)$

\section{Eosinophilic polyps vs. neutrophilic polyps}

The expressions of NOS-2 in the endothelium and stroma of eosinophilic polyps were significantly stronger than in neutrophilic polyps. In contrast, neutrophilic pol-

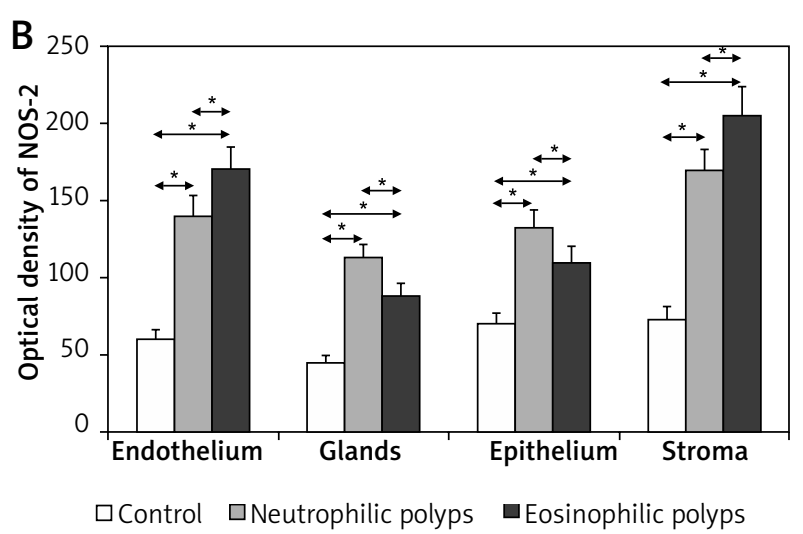

Figure 4. Expression of cyclooxygenase 2 (A) and nitric oxide synthase 2 (B) in selected structures of nasal mucosa. Statistically significant differences $(p<0.05)$ 
yps showed significantly higher levels of glandular and epithelial expressions of NOS-2.

\section{Discussion}

The modulation of ongoing inflammation is not completely understood, but likely involves a complex interplay between the cellular elements of the sinus tissue and the native and recruited immune cells.

We analyzed the differences in the intensity and topography of TNF- $\alpha, I L-1 \beta$, COX-2, NOS- 2 and NF- $\kappa$ B expressions in tissues of nasal polyps, representing various inflammatory phenotypes, and in chronic inflammatory nasal mucosa from patients with septal deviation - controls. To the best of our knowledge, this was the first direct comparison between the expressions of these proteins in eosinophilic and neutrophilic polyps of European patients. Noticeably, we analyzed the expressions in situ, not in polyp tissue cultures.

Our findings point to a potential involvement of TNF- $\alpha$ in the development of nasal polyps. While we observed an enhanced expression of this cytokine in polyp tissues, it was virtually undetectable in nasal mucosa from the controls. Although a strong expression of TNF- $\alpha$ was documented in both analyzed inflammatory phenotypes of polyps, its significantly higher levels were observed in eosinophilic polyps. An enhanced expression of this cytokine in nasal polyps was previously reported by other authors $[4,6,12]$. Understanding the role played by TNF- $\alpha$ in the development of nasal polyps seems vitally important as identification of its involvement in the pathogenesis of other conditions (e.g. rheumatoid arthritis, psoriasis) was reflected by successful attempts of their treatment with anti-TNF antibodies. Oyer et al. [13] presented increased adhesion molecules (vascular cell adhesion molecule 1 (VCAM-1) and intercellular adhesion molecule 1 (ICAM)) expression on sinonasal fibroblast in chronic sinusitis patients with and without nasal polyposis. Our observation that both analyzed phenotypes of polyps showed stronger expressions of IL-1 $\beta$ than control nasal mucosa is consistent with current knowledge on the functioning of this cytokine [14]. Plausibly, the enhanced expression of IL- $1 \beta$ reflected the proliferation associated with polyp growth and inflammatory processes taking place in polyp tissues. Zhang et al. [4] showed that concentration of IL-1 $\beta$ in homogenates of neutrophilic polyps from Chinese patients is significantly higher than in eosinophilic polyps of Belgian patients. The neutrophilic phenotype of inflammation in nasal polyps of patients from Asia was previously attributed to upregulation of IL-1 $\beta$ and the presence of Th17 lymphocytes. However, except for stromal cells for which the difference proved statistically insignificant, the expressions of this cytokine in eosinophilic polyp tissues were significantly stronger than in neutrophilic lesions. A study on mice revealed that, depending on the stage of the disease and its treatment, interactions may occur in the airways between Th2-type immune response with eosinophilia and Th17-type response with neutrophilia [15]. It should be remembered that IL-1 $\beta$ plays an established role in protection against bacterial and fungal infections [16]. Furthermore, both IL-1 $\beta$ and TNF- $\alpha$ induce an endothelial expression of VCAM-1 in the microvessels of polyps; this is reflected by adhesion and transmigration of eosinophils, but not neutrophils $[1,17]$. In our study, the expressions of IL-1 $\beta$ and TNF- $\alpha$ turned out to be the strongest in vascular endothelium of the eosinophilic polyps. Therefore, it is likely that some patients with nasal polyps develop nasal eosinophilia secondarily to increased activities of IL-1 $\beta$ and TNF- $\alpha$. While eosinophils can play a role in the remodeling of nasal polyp tissues, their involvement in the development of these lesions is unlikely. If eosinophilia was involved in the pathogenesis of nasal polyposis, the prevalence of polyps in patients with allergic rhinitis would be markedly higher than documented in the epidemiological studies. It may be speculated that the relative deficit of $T$ regulatory cells accounts for the inability to suppress inflammation, resulting in a stronger inflammatory response in CRS with polyps compared with CRS patients.

Furthermore, the strong expression of NF- $\kappa \mathrm{B}$ observed in nasal polyps analyzed in our study represented a marker of enhanced inflammation. However, the expressions of this transcription factor in eosinophilic and neutrophilic polyps did not differ as evidently as the levels of IL-1 $\beta$ and TNF- $\alpha$. Significant interphenotypic differences pertained solely to epithelial and stromal expressions of NF- $\mathrm{KB}$, both stronger in eosinophilic polyps. A strong expression of NF- $\mathrm{KB}$ in polyp tissue and an inadequate dose of glucocorticoids were postulated as determinants of worse response to pharmacotherapy [18, 19]. It is noteworthy that all our patients were qualified to surgery due to failure of the pharmacological treatment of nasal polyposis.

Apart from being associated with inflammation, an enhanced expression of COX-2 can also promote tumorigenesis [20]. Altered regulation of arachidonic acid cascade metabolism is a well-documented feature of nasal polyps, and is observed irrespectively of tolerance to non-steroidal anti-inflammatory drugs [21]. However, the available data on the dynamics of COX-2 expression (at both the mRNA and protein (evel) are inconclusive. While, similarly to our study, some authors documented an up-regulation of COX-2 expression in polyp tissues [22-26], other researchers claimed a down-regulated expression of this enzyme $[27,28]$. The expression of COX-2 can be modulated by the severity of local inflammation, implemented treatment (e.g. inhibitory effect of glucocorticoids), and some comorbidities (e.g. allergies, aspirin-exacerbated respiratory disease); consequently, the expression of COX-2 in a given material reflects the inclusion criteria to a studied group. Moreover, the methodology of determining the COX-2 ex- 
pression (immunohistochemistry or PCR) can affect the results. The observation that analyzed phenotypes differed in terms of the distribution of peak levels of COX-2 expression is an original contribution of our study. Compared to neutrophilic lesions, eosinophilic polyps were characterized by a significantly stronger expression of COX-2 in glandular and stromal cells. In contrast, the epithelial and endothelial expression of this enzyme was stronger in the case of neutrophilic polyps. The quantitative determination of mediators concentration in the tissue (qRT-PCR analysis) would be a valuable complement to our observations. Our observations regarding eosinophilic polyps are partially consistent with the data published by Gosepath et al. [29]. They documented a strong glandular expression of COX-2 and weak epithelial expression of this enzyme in polyps of patients with concomitant allergy or aspirin intolerance (and thus, probably, in eosinophilic polyps) [29]. Understanding the role of overexpression of this enzyme in the development of polyps representing various inflammatory phenotypes is also important in the context of their treatment, not necessarily solely with glucocorticoids. For example, inhibitors of COX-2 are used in pharmacological prevention of colorectal cancer in individuals with familial polyposis [30]. It has been observed that the COX-2 expression level was higher in a statistically significant manner in the mucosa of patients with CRS without polyps as compared to the expression level in the mucosa and polyp tissue in patients with CRS with polyps [31]. Elevated levels of oral and nasal FeNO (fractional concentration of exhaled nitric oxide) were shown to be associated with overexpression of NOS-2, and are specific for eosinophilic chronic rhinosinusitis [32]. Parikh et al. [33] observed that the activity of NOS-2 in eosinophilic polyps of patients with asthma and aspirin sensitivity was significantly higher than in nasal polyps of persons without asthma or with asthma without aspirin sensitivity. We observed an overexpression of NOS-2 in tissues of both eosinophilic and neutrophilic polyps. The endothelial and stromal expressions of NOS-2 were significantly stronger in eosinophilic than in neutrophilic polyps. In contrast, glandular and epithelial expressions of NOS-2 turned out to be significantly stronger in neutrophilic polyps. This suggests that macrolide antibiotics and fexofenadine hydrochloride, both significantly reducing the airway synthesis of nitric oxide, could be used as an adjuvant to glucocorticoids in the pharmacotherapy of nasal polyps, especially of their non-eosinophilic phenotype [34-36]. Our results contribute to a growing body of literature showing a locally elevated expression of inflammatory mediators in patients with chronic rhinosinusitis and polyps.

\section{Conclusions}

The NF- $\kappa B, I L-1 \beta$, TNF- $\alpha$, COX-2 and NOS-2 seem to be involved in the pathogenesis of nasal polyps, irrespective of their phenotype. The lack of major differences be- tween eosinophilic and neutrophilic polyps suggests the involvement of common pathogenic pathways in their development. The differences in the intensity of COX-2 and NOS-2 expressions could reflect various stages of the inflammatory process and the involvement of various cells in the pathogenesis of eosinophilic and neutrophilic polyps. The expressions of most markers were the strongest in vascular endothelium of both phenotypes, which suggests that endothelial defects can play an important role in the pathogenesis of nasal polyps.

\section{Conflict of interest}

The authors declare no conflict of interest.

\section{References}

1. Hamilos DL, Leung DY, Wood R, et al. Eosinophil infiltration in nonallergic chronic hyperplastic sinusitis with nasal polyposis (CHS/NP) is associated with endothelial VCAM-1 upregulation and expression of TNF-alpha. Am J Respir Cell Mol Biol 1996; 15: 443-50.

2. Cao PP, Li HB, Wang BF, et al. Distinct immunopathologic characteristics of various types of chronic rhinosinusitis in adult Chinese. J Allergy Clin Immunol 2009; 124: 478-84, 84 e1-2.

3. Fokkens WJ, Lund VJ, Mullol J, et al. EPOS 2012: European position paper on rhinosinusitis and nasal polyps 2012. A summary for otorhinolaryngologists. Rhinology 2012; 50: 1-12.

4. Zhang N, Van Zele T, Perez-Novo C, et al. Different types of T-effector cells orchestrate mucosal inflammation in chronic sinus disease. J Allergy Clin Immunol 2008; 122: 961-8.

5. Dayer JM. Interleukin 1 or tumor necrosis factor-alpha: which is the real target in rheumatoid arthritis? I Rheumatol 2002; Suppl. 65: 10-5.

6. Hori M, Kita M, Torihashi S, et al. Upregulation of iNOS by COX-2 in muscularis resident macrophage of rat intestine stimulated with LPS. Am J Physiol Gastrointest Liver Physiol 2001; 280: G930-8.

7. Kim SF. The role of nitric oxide in prostaglandin biology: update. Nitric Oxide 2011; 25: 255-64.

8. Kuwano T, Nakao S, Yamamoto H, et al. Cyclooxygenase 2 is a key enzyme for inflammatory cytokine-induced angiogenesis. FASEB J 2004; 18: 300-10.

9. Cho JS, Kang JH, Shin JM, et al. Inhibitory effect of delphinidin on extracellular matrix production via the MAPK/NFkappaB pathway in nasal polyp-derived fibroblasts. Allergy Asthma Immunol Res 2015; 7: 276-82.

10. Plewka A, Madej P, Plewka D, et al. Immunohistochemical localization of selected pro-inflammatory factors in uterine myomas and myometrium in women of various ages. Folia Cytochem Cytobiol 2013; 51: 73-83.

11. Read N, Rhodes P. Techniques for image analysis. In: Immunocytochemistry. A practical approach. Beesley J (ed.). Oxford University Press, Oxford 1993; 127-49.

12. Oyer SL, Mulligan JK, Psaltis AJ, et al. Cytokine correlation between sinus tissue and nasal secretions among chronic rhinosinusitis and controls. Laryngoscope 2013; 123: 72-8.

13. Oyer SL, Nagel W, Mulligan JK. Differential expression of adhesion molecules by sinonasal fibroblasts among control and chronic rhinosinusitis patients. Am J Rhinol Allergy 2013; 27: 381-6. 
14. van de Veerdonk FL, Netea MG. New insights in the immunobiology of IL-1 family members. Front Immunol 2013; 4: 167-9.

15. Besnard AG, Togbe D, Couillin I, et al. Inflammasome-IL1-Th17 response in allergic lung inflammation. J Mol Cell Biol 2012; 4: 3-10.

16. Mills KH, Dungan LS, Jones SA, Harris J. The role of inflammasome-derived IL-1 in driving IL-17 responses. J Leukoc Biol 2012; 93: 489-97.

17. Bachert C, Gevaert P, Holtappels G, van Cauwenberge P. Mediators in nasal polyposis. Curr Allergy Asthma Rep 2002; 2: 481-7.

18. Grzanka A, Misiolek M, Golusinski W, Jarzab J. Molecular mechanisms of glucocorticoids action: implications for treatment of rhinosinusitis and nasal polyposis. Eur Arch Otorhinolaryngol 2011; 268: 247-53.

19. Valera FC, Scrideli C, Queinoz R, et al. NF-kappaB expression predicts clinical outcome for nasal polyposis. Rhinology 2010; 48: 408-41.

20. Chen YF, Luo RZ, Li Y, et al. High expression levels of COX-2 and P300 are associated with unfavorable survival in laryngeal squamous cell carcinoma. Eur Arch Otorhinolaryngol 2013; 270: 1009-17.

21. Pawliczak R, Lewandowska-Polak A, Kowalski ML. Pathogenesis of nasal polyps: an update. Curr Allergy Asthma Rep 2005; 5: 463-71.

22. Fernandez-Morata JC, Mullol J, Fuentes M, et al. Regulation of cyclooxygenase- 1 and -2 expression in human nasal mucosa. Effects of cytokines and dexamethasone. Clin Exp Allergy 2000; 30: 1275-84.

23. Liu CM, Hong CY, Shun CT, et al. Inducible cyclooxygenase and interleukin 6 gene expressions in nasal polyp fibroblasts: possible implication in the pathogenesis of nasal polyposis. Arch Otolaryngol Head Neck Surg 2002; 128: 945-51.

24. Owens JM, Shroyer KR, Kingdom TT. Expression of cyclooxygenase and lipoxygenase enzymes in nasal polyps of aspirin-sensitive and aspirin-tolerant patients. Arch Otolaryngo Head Neck Surg 2006; 132: 579-87.

25. Roca-Ferrer J, Pujols L, Gartner S, et al. Upregulation of COX-1 and COX-2 in nasal polyps in cystic fibrosis. Thorax 2006; 61: 592-6.

26. Yun CB, Lee BH, Jang TJ. Expression of glucocorticoid receptors and cyclooxygenase-2 in nasal polyps from nonallergic patients. Ann Otol Rhinol Laryngol 2002; 111: 61-7.

27. Picado C, Fernandez-Morata JC, Juan M, et al. Cyclooxygenase-2 mRNA is downexpressed in nasal polyps from aspirinsensitive asthmatics. Am J Respir Crit Care Med 1999; 160: 291-6.

28. Roca-Ferrer J, Garcia-Garcia FJ, Pereda J, et al. Reduced expression of COXs and production of prostaglandin $\mathrm{E}(2)$ in patients with nasal polyps with or without aspirin-intolerant asthma. J Allergy Clin Immunol 2011; 128: 66-72.

29. Gosepath J, Brieger J, Gletsou E, Mann WJ. Expression and localization of cyclooxygenases (Cox-1 and Cox-2) in nasal respiratory mucosa. Does Cox-2 play a key role in the immunology of nasal polyps? I Investig Allergol Clin Immunol 2004; 14: 114-8.

30. Higuchi T, Iwama T, Yoshinaga $\mathrm{K}$, et al. A randomized, double-blind, placebo-controlled trial of the effects of rofecoxib, a selective cyclooxygenase- 2 inhibitor, on rectal polyps in familial adenomatous polyposis patients. Clin Cancer Res 2003; 9: 4756-60.

31. Miloński J, Zielinska-Blizniewska H, Przybyłowska K, et al. Significance of cyclooxygenase-2 (COX-2), periostin (POSTN) and interleukin-4 (IL-4) gene expression in the pathogenesis of chronic rhinosinusitis with nasal polyps. Eur Arch Otorhinolaryngol 2015; 272: 3715-20.

32. Takeno S, Taruya T, Ueda T, et al. Increased exhaled nitric oxide and its oxidation metabolism in eosinophilic chronic rhinosinusitis. Auris Nasus Larynx 2013; 40: 458-64.

33. Parikh A, Scadding GK, Gray P, et al. High levels of nitric oxide synthase activity are associated with nasal polyp tissue from aspirin-sensitive asthmatics. Acta Otolaryngol 2002; 122: 302-5.

34. Asano K, Kamakazu K, Hisamitsu T, Suzaki H. Suppressive activity of macrolide antibiotics on nitric oxide production from nasal polyp fibroblasts in vitro. Acta Otolaryngol 2003; 123: 1064-9.

35. Asano K, Kanai K, Furuta A, et al. Suppressive activity of fexofenadine hydrochloride on nitric oxide production invitro and in-vivo. J Pharm Pharmacol 2007; 59: 1389-95.

36. Haruna S, Shimada C, Ozawa M, et al. A study of poor responders for long-term, low-dose macrolide administration for chronic sinusitis. Rhinology 2009; 47: 66-71. 